\section{Fusarium incarnatum/equiseti hemodialysis graft infection}

\author{
James Riddell IV, \\ Kenneth J. Woodside, ${ }^{2 *}$ \\ Matthew A. Leavitt, ${ }^{3}$ Duane W. Newton, ${ }^{4}$ \\ Jeffery D. Punch ${ }^{2}$ \\ 'Department of Internal Medicine, \\ Division of Infectious Diseases; \\ 2Department of Surgery, Division \\ of Transplantation, "Current address: \\ Department of Surgery, Division of \\ Transplant and Hepatobiliary Surgery, \\ University Hospitals, Case Medical \\ Center; ${ }^{3}$ Department of Internal Medicine, \\ Division of Nephrology; ${ }^{4}$ Department of \\ Pathology, Clinical Microbiology \\ Laboratory, University of Michigan \\ Medical School, Michigan, USA
}

\begin{abstract}
Hemodialysis graft infections typically occur as a result of contamination by skin flora at the time of insertion or become secondarily infected after high-grade bacteremia. Infection of implanted vascular devices with filamentous fungi is rare. We report a case of infection of an implanted polytetrafluoroethylene dialysis graft with Fusarium incarnatum/equiseti that did not grow in cultures of tissue but was identified by molecular means.
\end{abstract}

\section{Case Report}

A 58-year-old woman with Crohn's disease, nephrolithiasis, and chronic renal insufficiency secondary to obstructive nephropathy required placement of a dialysis graft prior to the initiation of hemodialysis. The procedure was performed in a tertiary care hospital operating room. She was not receiving immunosuppressive agents for her Crohn's disease. Within 48 hours after placement of the graft she developed yellowish drainage, pain, erythema, and swelling at the distal incision just proximal to the antecubital fossa. She received multiple courses of treatment with oral cephalexin and IV vancomycin, without improvement in her symptoms. Over a period of a month she developed an enlarging, firm, nodular lesion at the site of her distal incision (Figure 1). She subsequently underwent surgical exploration and debridement. The graft was found to be poorly incorporated and was removed, as was the firm cyst-like lesion (Figure 2: histopathology). Fungal cultures of the tissue and graft were sterile. Fusarium incarnatum/equiseti species complex DNA was detected with a $28 \mathrm{~S}$ primer set by the University of Washington Department of Laboratory Medicine from fresh tissue (not fixed in formalin).

\section{Discussion}

Fusarium is a ubiquitous mold found in soil and water and is a common pathogen of plants and stored grains. It is considered to be an agent of hyalohyphomycosis, which is defined as a human infection with a saprophytic mold with hyaline, branched or unbranched, hyphal elements without the presence of pigment in the cell wall. ${ }^{1}$ Other common species in this group include Aspergillus, Scopulariopsis, Pseudallescheria, and Scedosporium. There are over 50 species contained within the Fusarium genus. The species most commonly associated with human disease are Fusarium solani (50\%), F. oxysporum (20\%), and F. verticillioides (10\%). ${ }^{2}$ Other less common species include Fusarium chlamydosprum, F. dimerum, as well as $F$. incarnatum/equiseti.

Fusarium species most often cause infection in immune compromised hosts such as stem cell transplant recipients or patients with prolonged neutropenia. ${ }^{2,3}$ The usual portal of entry is the respiratory route versus direct inoculation of cutaneous structures or foreign bodies such as central venous catheters. Sinopulmonary disease and subsequent dissemination can occur in susceptible patients. In contrast to other filamentous fungi, Fusarium can be cultured from the blood in cases of disseminated disease. The dermatological manifestations of disseminated fusariosis consist of subcutaneous nodules or ecthyma-like skin lesions occasionally observed with a targetoid appearance with surrounding erythema. Other clinical syndromes include keratitis associated with contaminated contact lenses, endophthalmitis, onychomycosis, cutaneous infections from direct inoculation, and catheter/foreign body-associated infection. Fusarium infection of a dialysis access graft has not been reported previously. In our case, it is unknown how the implanted polytetra fluoroethylene dialysis graft became contaminated. The device had not yet been accessed for hemodialysis and no clinical evidence of disseminated disease was identified elsewhere. Thus, the device itself could have been contaminated prior to, or at the time of surgical implantation. No other cases of procedurerelated invasive fungal infections were reported from the operating suite by the hospital's infection control surveillance team.

The cornerstone of treatment for patients with localized infection or infection of foreign bodies is surgical debridement, with removal of
Correspondence: James Riddell IV, 1500 E Medical Center Drive, 3120 Taubman Center, Ann Arbor, MI 48109-5378, USA.

E-mail: jriddell@umich.edu

Key words: Fusarium incarnatum, Fusarium equiseti, infection, hemodialysis, graft.

Contributions: all authors managed the patient; JR authored the manuscript; KW, ML, DN, JP reviewed the manuscript; JP obtained the image for Figure 1; DN obtained Figure 2 and managed the molecular testing.

Acknowledgments: JP has received grants from Roche, Novartis, Viropharma, and Astellas.

Received for publication: 24 June 2010.

Revision received: 17 September 2010.

Accepted for publication: 17 September 2010

This work is licensed under a Creative Commons Attribution 3.0 License (by-nc 3.0).

(C) Copyright J.Riddell et al., 2010

Licensee PAGEPress, Italy

Infectious Disease Reports 2010; 2:e14

doi:10.4081/idr.2010.e14

all infected material, particularly any foreign bodies such as surgical implants. Antifungal therapy is also important in localized infection as well as disseminated disease. The best antifungal agent for the treatment of Fusarium infection has not been defined. For the most common Fusarium species: F. solani and F. ver-

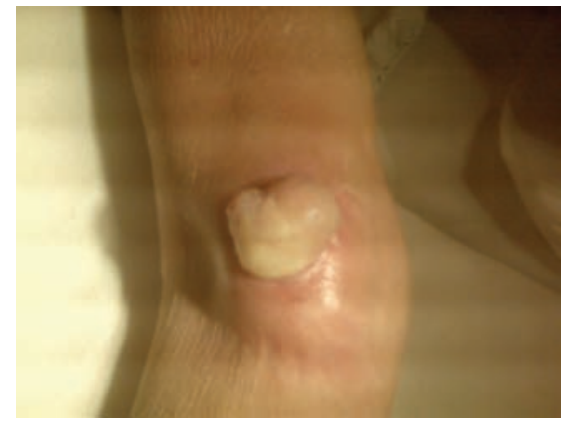

Figure 1. Appearance of the right antecubital fossa prior to surgical resection.

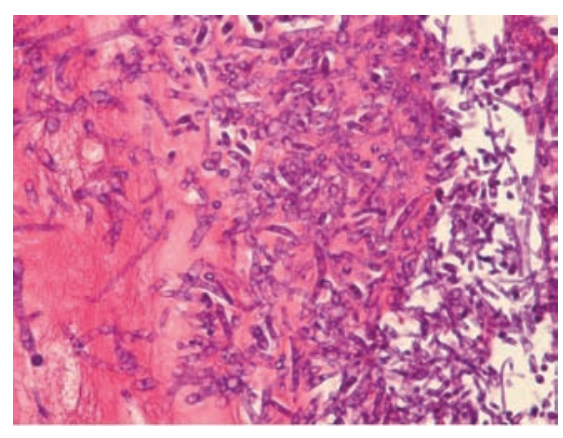

Figure 2. Hematoxylin and eosin stain of resected tissue (magnification $400 \mathrm{X}$ ). 
ticullioides, an amphotericin B preparation is considered to be the treatment of choice by some authors. ${ }^{2}$ In vitro studies have shown that MICs to voriconazole and posaconazole are $>8.0 \mu \mathrm{g} / \mathrm{mL}$ in these species while amphotericin B susceptibility is preserved in the 1.0 $\mu \mathrm{g} / \mathrm{mL}$ range. ${ }^{4,5}$ For the less common species such as $F$. incarnatum/equisiti there is less data. In a study that evaluated in vitro susceptibilities of this species from nine clinical isolates from India and Chile, the voriconazole MIC was 2-4 $\mu \mathrm{g} / \mathrm{mL}$ with comparable posaconazole MICs. ${ }^{6}$ Therefore, some experts have concluded that if $F$. solani or $F$. verticillioides is identified, high-dose amphotericin $B$ should be used, and for other species amphotericin B and/or voriconazole can be administered. ${ }^{2}$

Currently, voriconazole is the only drug that is FDA approved for treatment of Fusarium sp. in the United States. In licensing trials for voriconazole, out of 21 patients with invasive Fusarium infection, 9 or $43 \%$ had a favorable response to voriconazole. ${ }^{7}$ Three of the nine patients who had a successful outcome had an infection with $F$. solani. No other randomized trials or large case series are available. One group has reported a breakthrough infection with resistant Fusarium sp. while on treatment with voriconazole. ${ }^{8}$ However, many other published case reports have described successful treatment with voriconazole in cases of soft tissue infection, disseminated infection, and endophthalmitis. ${ }^{9-14}$ Successful outcomes utilizing combination therapy consisting of voriconazole with amphotericin B products have also been reported in similar cases. ${ }^{15-19}$ Less experience is available regarding the use of posaconazole; however, this may also be an effective drug. ${ }^{20-22}$ The echinocandins have been found to have high MICs to Fusarium ( $>16 \mu \mathrm{g} / \mathrm{mL}$ ) and thus should not be used for primary treatment. However, synergy of echinocandins with amphotericin B has been demonstrated in vitro. ${ }^{23}$

In our case, voriconazole rather than an amphotericin B product was used, as the patient had stage IV-V chronic kidney disease and was at high risk for the need for hemodialysis. Initial treatment was complicated by transaminitis, with a measured plasma voriconazole level of $3.8 \mu \mathrm{g} / \mathrm{mL}$. The dose was decreased to achieve levels of $1.4-1.9 \mu \mathrm{g} / \mathrm{mL}$, with resolution of liver enzyme elevation. After six months of therapy, the surgical wound had completely healed and treatment with voriconazole was discontinued. This case illustrates the benefits of molecular testing to identify pathogens that are difficult to grow. Identifying clinical Fusarium isolates to the species level has important implications for the selection of an appropriate therapeutic regimen.

\section{References}

1. Dismukes W, Pappas P, Sobel J. Clinical Mycology. Oxford: Oxford University Press, 2003, pp 252-70.

2. Nucci M, Anaissie E. Fusarium infections in immunocompromised patients. Clin Microbiol Rev 2007;20:695-704.

3. Dignani MC, Anaissie E. Human fusariosis. Clin Microbiol Infect 2004;10:67-75.

4. Cuenca-Estrella M, Gomez-Lopez A, Mellado E, et al. Head-to-head comparison of the activities of currently available antifungal agents against 3,378 Spanish clinical isolates of yeasts and filamentous fungi. Antimicrob Agents Chemother 2006;50:917-21.

5. Tortorano AM, Prigitano A, Dho G, et al. Species distribution and in vitro antifungal susceptibility patterns of 75 clinical isolates of Fusarium spp. from northern Italy. Antimicrob Agents Chemother 2008;52:2683-5.

6. Azor M, Gene J, Cano J, et al. Less-frequent Fusarium species of clinical interest: correlation between morphological and molecular identification and antifungal susceptibility. J Clin Microbiol 2009; 47:1463-8.

7. Pfizer. Voriconazole package insert. 2008.

8. Cudillo L, Girmenia C, Santilli S, et al. Breakthrough fusariosis in a patient with acute lymphoblastic leukemia receiving voriconazole prophylaxis. Clin Infect Dis 2005;40:1212-3.

9. Consigny S, Dhedin N, Datry A, Choquet S, Leblond V, Chosidow O. Successful voriconazole treatment of disseminated Fusarium infection in an immunocompromised patient. Clin Infect Dis 2003;37:3113.

10. Durand ML, Kim IK, D'Amico DJ, et al. Successful treatment of Fusarium endophthalmitis with voriconazole and Aspergillus endophthalmitis with voriconazole plus caspofungin. Am J Ophthalmol 2005;140: $552-4$.

11. Bigley VH, Duarte RF, Gosling RD, et al. Fusarium dimerum infection in a stem cell transplant recipient treated successfully with voriconazole. Bone Marrow Transplant 2004;34:815-7.

12. Guimera-Martin-Neda F, Garcia-Bustinduy M, Noda-Cabrera A, et al. Cutaneous infection by Fusarium: successful treatment with oral voriconazole. Br J Dermatol 2004; 150:777-8.

13. Reis A, Sundmacher R, Tintelnot K, et al. Successful treatment of ocular invasive mould infection (fusariosis) with the new antifungal agent voriconazole. $\mathrm{Br} \mathrm{J}$ Ophthalmol 2000;84:932-3.

14. Vincent AL, Cabrero JE, Greene JN, Sandin RL. Successful voriconazole therapy of disseminated Fusarium solani in the brain of a neutropenic cancer patient. Cancer Control 2003;10:414-9.

15. Rodriguez CA, Lujan-Zilbermann J, Woodard P, et al. Successful treatment of disseminated fusariosis. Bone Marrow Transplant 2003;31:411-2.

16. Guzman-Cottrill JA, Zheng X, Chadwick EG. Fusarium solani endocarditis successfully treated with liposomal amphotericin B and voriconazole. Pediatr Infect Dis J 2004;23:1059-61.

17. Durand-Joly I, Alfandari S, Benchikh Z, et al. Successful outcome of disseminated Fusarium infection with skin localization treated with voriconazole and amphotericin B-lipid complex in a patient with acute leukemia. J Clin Microbiol 2003;41: 4898-900.

18. Stanzani M, Vianelli N, Bandini G, et al. Successful treatment of disseminated Fusariosis after allogeneic hematopoietic stem cell transplantation with the combination of voriconazole and liposomal amphotericin B. J Infect 2006;53:e243-6.

19. Ho DY, Lee JD, Rosso F, Montoya JG. Treating disseminated fusariosis: amphotericin $\mathrm{B}$, voriconazole or both? Mycoses 2007;50:227-31.

20. Tu EY, McCartney DL, Beatty RF, et al. Successful treatment of resistant ocular fusariosis with posaconazole (SCH56592). Am J Ophthalmol 2007;143:222-7.

21. Gupta S, Almyroudis NG, Battiwalla M, et al. Successful treatment of disseminated fusariosis with posaconazole during neutropenia and subsequent allogeneic hematopoietic stem cell transplantation. Transpl Infect Dis 2007;9:156-60.

22. Raad II, Hachem RY, Herbrecht R, et al. Posaconazole as salvage treatment for invasive fusariosis in patients with underlying hematologic malignancy and other conditions. Clin Infect Dis 2006;42:1398403.

23. Arikan S, Lozano-Chiu M, Paetznick V, Rex JH. In vitro synergy of caspofungin and amphotericin B against Aspergillus and Fusarium spp. Antimicrob Agents Chemother 2002;46:245-7. 